\title{
Population Dynamics of Thrips tabaci Lindeman (Thysanoptera: Thripidae) on Table Grapes
}

\author{
A. Schwartz \\ Viticultural and Oenological Research Institute, Private Bag X5026, Stellenbosch, Republic of South Africa. \\ Submitted for publication: March 1988 \\ Accepted for publication: April 1988 \\ Keywords: Thrips, table grapes, population dynamics, grapevine.
}

\begin{abstract}
Young vegetative shoots of three table grape cultivars were infested by adults of Thrips tabaci during spring. The infestation on clusters of flowers and young fruit, which followed was brief. Maximum numbers of $\boldsymbol{T}$. tabaci were recorded during the blossom stage. Shoots and leaves were infested by $\boldsymbol{T}$. tabaci for the duration of the season. Several natural enemies occurred with the $T$. tabaci populations on the shoots and leaves of which Amblyseius addoensis Van der Merwe \& Ryke, a predaceous mite, was the most numerous.
\end{abstract}

Thrips have been known to be associated with the flowers of grapevines for many years, but only recently have isolated cases of damage to table grapes been noted in the Western Cape. The damage consisted of a scarring of the berry skin. Scarred berries have to be removed before harvest and during packing, which is time consuming and may result in straggly bunches. In California, table grapes may be damaged by the thrips Frankliniella occidentalis (Pergande), resulting in scarred fruit. It is, however, considered a minor economic pest (Flaherty et al., 1982).

A survey in the Paarl and Hex River Valley indicated the presence of six species of thrips on the flowers of table grape cultivars, viz. Thrips tabaci Lindeman, Aeolothrips brevicornis Bagnall, Glaucothrips glaucus Bagnall, Heliothrips sylvanus Faure, Heliothrips haemorrhoidalis Bouché and a Haplothrips sp. (identified by Jenny Palmer, British Museum). Thrips tabaci was by far the dominant species (70-100\%; unpublished data) and hold the potential for damage to the young developing berries.

In order to establish the critical time of damage to the young berry, it was necessary to study the population dynamics of $T$. tabaci on the vine. This in turn would determine the scheduling of chemical control measures.

\section{MATERIALS AND METHODS}

Sampling of post-budding shoots and leaves from developed shoots

Sampling in the Paarl area was conducted in each of three vineyards, i.e. cultivars Waltham Cross, Queen of the Vineyard and Barlinka during the 1984/85 season. The vineyards were subjected to a standard spray programme for disease control.

Sampling commenced at the bud burst stage and was initially carried out once a week. One new shoot, including the leaves, from a single vine for each of five plots in a row and for 10 rows, i.e. a total of 50 shoots per vineyard, was removed according to the method by Schwartz (1987). This is known as the alcohol-wash method. The five shoots from one row were sampled into a single jar containing a 70 percent alcohol solution, ending with 10 jars per date.

As soon as the shoots with leaves had developed suf- ficiently, the sampling schedule was lengthened to biweekly intervals and adapted to consist of leaves from three positions on a shoot. The terminal $150 \mathrm{~mm} \mathrm{sec-}$ tion of the shoot with attached leaves, one middle position leaf and one basal leaf was cut into a jar containing the 70 percent alcohol solution. Immediately following sampling the contents of the jar were shaken to dislodge all organisms from the leaves. The leaves were removed with a forceps and the jars taken to the laboratory. After standing overnight the excess supernatent was drawn off and the rest containing the precipitant poured into a petri dish. All motile stages of $T$. tabaci were counted. Natural enemies were also noted.

\section{Sampling of flowers and young fruit clusters}

From flowering the aforementioned plots in each of the three vineyards were sampled at weekly intervals. Two flower clusters (later : fruit clusters) per plant were examined for a total of 100 . The flower or fruit clusters were struck with the palm of the hand while a tray $(370 \times 250 \mathrm{~mm})$ with a white surface was held underneath. The thrips were dislodged and dropped onto the tray where they were counted. Observations in the Barlinka vineyard were also carried out during the $1985 / 86$ season.

\section{RESULTS AND DISCUSSION}

Seasonal occurrence of $T$. tabaci on young shoots and leaves from developed shoots

The first young shoots, following bud burst, became infested with adult $T$. tabaci almost immediately. It is assumed that these adults had emerged from overwintering pupae in the soil. Two weeks later the first of the young thrips larvae emerged and were present with the adults. Whereas Waltham Cross and Queen of the Vineyard reached the bud burst stage at about the same time (21st August), Barlinka, being a late cultivar, responded one month later.

During the season two periods of abundance could be distinguished for the thrips population, viz. August to November and January to March. Differences regarding peak populations were evident for the three cultivars. Thus peak populations for adults on Waltham Cross occurred during October and February (Fig. 1A). For Queen of the Vineyard peaks were recorded during August and February (Fig. 1B) and for Barlinka 

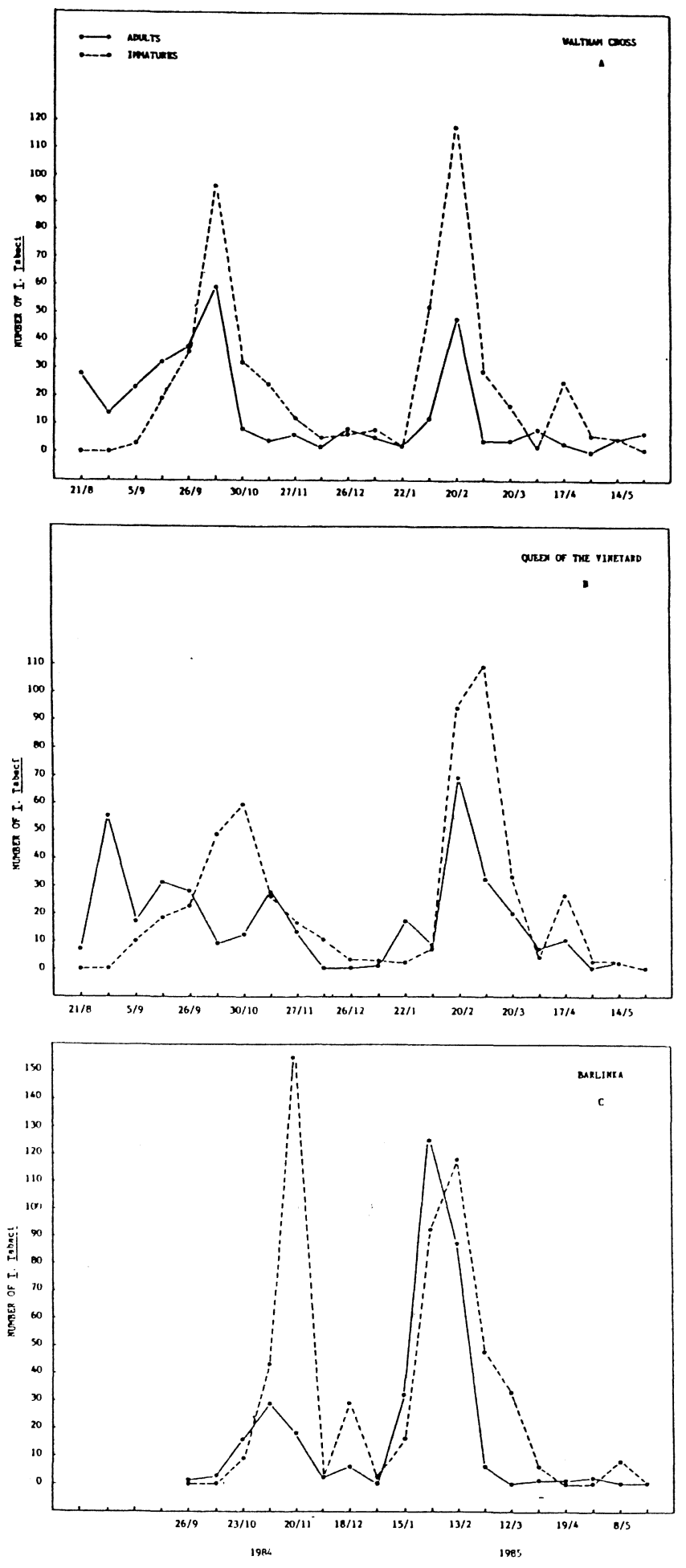

FIG. 1

Seasonal occurrence of Thrips tabaci on post budding shoots and leaves from developed shoots of Waltham Cross, Queen of the Vineyard and Barlinka, Paarl.

during November and January (Fig. 1C). This phenomenon can be ascribed largely to growth flush incidence as $T$. tabaci has a preference for young succulent leaves.

Peak populations for immature thrips on Waltham Cross corresponded with those of the adults, i.e. October and February. In the case of Queen of the Vine- yard population peaks for immature thrips occurred during October and March. Barlinka carried maximum numbers of immature thrips during November and February.

Populations of adult and immature thrips were generally at a low level of abundance during December and again from March onwards. None of the T. tabaci populations caused any discernible damage to the shoots or leaves.

Various natural enemies occurred together with the T. tabaci populations, viz. predators from the families Reduviidae (Order : Hemiptera) and Chrysopidae (Order : Neuroptera) as well as a predaceous thrips. However, they were few and sporadic in occurrence with their effect on $T$. tabaci unknown. A more prominent predator was Amblyseius addoensis Van der Merwe \& Ryke (Acarina : Phytoseiidae) which preyed upon the young larvae of $T$. tabaci (Schwartz, 1987). It was concluded that it did not succeed in preventing the build-up of the thrips population. However, it is envisaged that the survival and role of the predaceous mite may be enhanced by the use of selective spray chemicals. Moreover, the value of $A$. addoensis as control agent should ultimately be judged in the light of the economic threshold, a criterion which has not yet been established for T. tabaci on table grapes.

\section{Occurrence of $\boldsymbol{T}$. tabaci on flower and young fruit clus-} ters

On Waltham Cross T. tabaci was most abundant during the flowering stage (23rd October to 6th November prior to shedding of the "caps" (calyptra) (Fig. 2). The population subsequently dropped sharply and almost disappeared from the fruit clusters after a period of three weeks. On Queen of the Vineyard the T. tabaci population maintained relatively high numbers on two occasions seven days apart (23rd to 30th October) and thereafter drastically declined to very low numbers.

Observations on Barlinka indicated high numbers for $T$. tabaci on flower and fruit clusters for two sampling dates one week apart. Thereafter the population disappeared four weeks later (Fig. 3). Barlinka (characteristically) flowered later than Waltham Cross and Queen of the Vineyard.

The $T$. tabaci populations on the flower and fruit clusters did not cause lesions on the young berries. No natural enemies occurred with the $T$. tabaci populations on flower and young fruit clusters.

\section{CONCLUSIONS}

From the results obtained it is clear that the young vegetative shoots which appeared during spring were the first to be populated by $T$. tabaci. The population on these parts served as the source for the subsequent infestation of flowers. The duration of the thrips infestation of the flower and young fruit clusters was brief with maximum numbers recorded during the flowering stage. This is the critical time when damage through feeding is done to the young berries and appears to be closely associated with the shedding of the calyptra. The early flowering stage, therefore, would be the appropriate time for the application of chemical control measures.

The infestation of young shoots and leaves on developed shoots of three table grape cultivars by $T$. tabaci, 


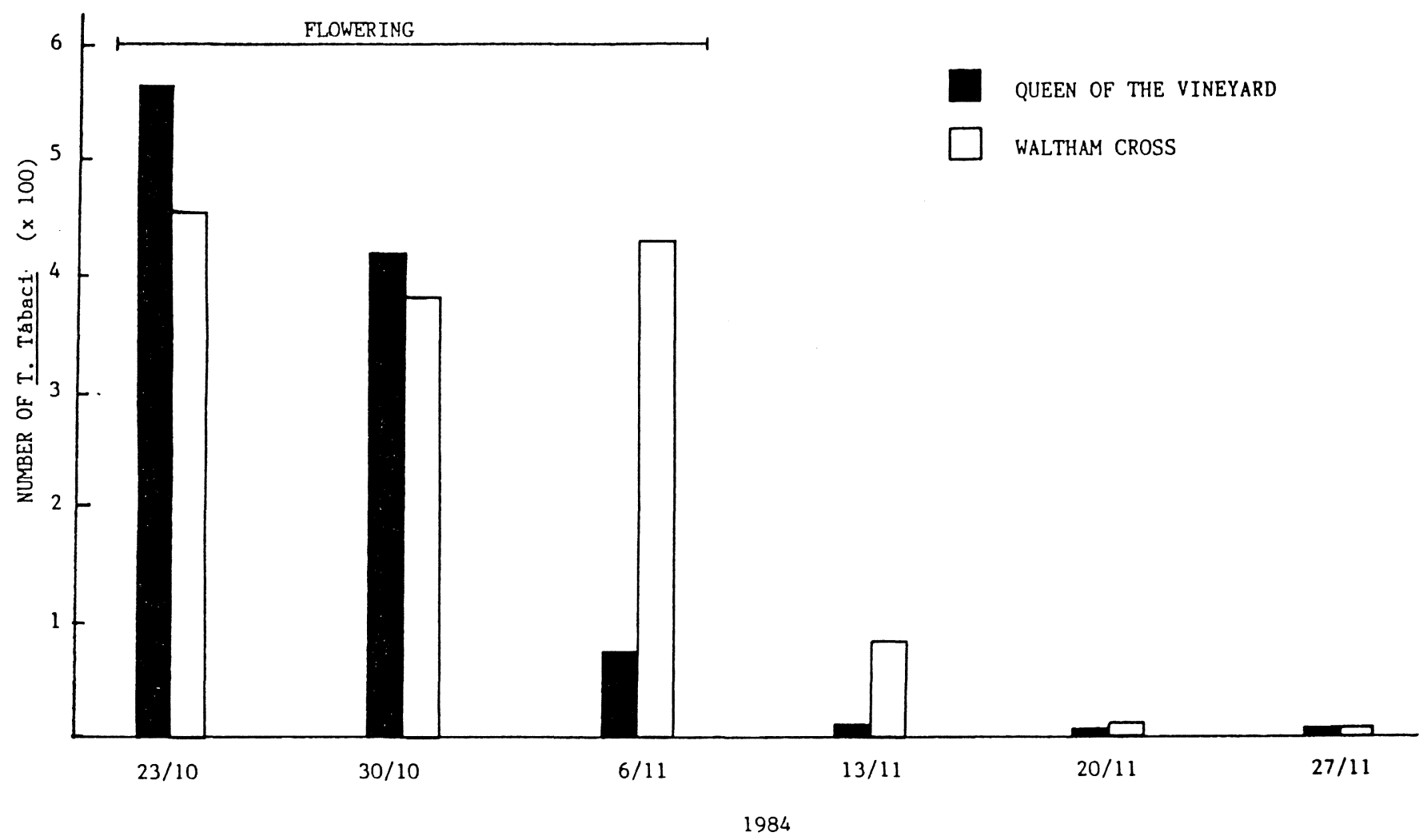

SAMPLING DATE

FIG. 2

Seasonal occurrence of Thrips tabaci on flower and fruit clusters of Waltham Cross and Queen of the Vineyard, Paarl

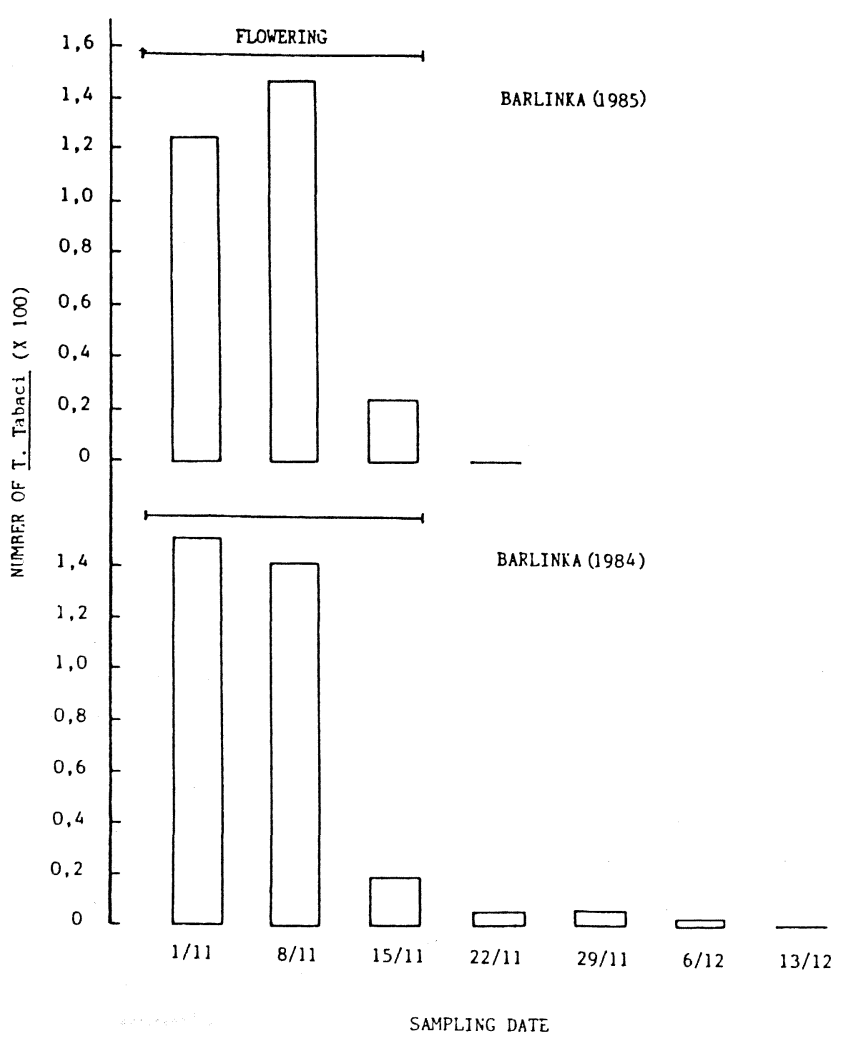

FIG. 3

Seasonal occurrence of Thrips tabaci on flower and fruit clusters of Barlinka, Paarl. lasted throughout the season. Although population peaks were noted, these remained at non-damaging levels. This phenomenon could possibly be ascribed to the influence exerted by climatic conditions and/or natural enemies. Several natural enemies, of which $A$. addoensis was the most numerous and consistent, were found in assosiation with the $T$. tabaci populations on the shoots and leaves of the plants.

\section{LITERATURE CITED}

FLAHERTY, D.L., JENSEN, F.L., KASIMATIS, A.N., KIDO, H. \& MOLLER, W.J., 1982. Grape pest management. In : Jensen, F.L., Flaherty, D.L. \& Luvisi, A. (eds.). Major insect and mite pests. Agricultural Sciences Publications, University of $\mathrm{Ca}$ lifornia, Berkeley. 97-190.

SCHWARTZ, A., 1987. Seasonal occurrence of a predaceous mite Amblyseius addoensis Van der Merwe \& Ryke (Acari : Phytoseiidae) on table grapes. S. Afr. J. Enol. Vitic. 8, 78-79. 